\title{
Effect of Induced Visual Fatigue on Sensory Ocular Dominance and the Effect of Colored Filters on the Levels of Visual Fatigue in Dyslexic Adults with MIS
}

\author{
Mana Alafri Alanazi \\ Department of Optometry, College of Applied Medical Sciences, King Saud University, Riyadh, Saudi Arabia \\ Email address: \\ amana@ksu.edu.sa \\ To cite this article: \\ Mana Alafri Alanazi. Effect of Induced Visual Fatigue on Sensory Ocular Dominance and the Effect of Colored Filters on the Levels of \\ Visual Fatigue in Dyslexic Adults with MIS. International Journal of Ophthalmology \& Visual Science. Vol. 5, No. 1, 2020, pp. 1-10. \\ doi: 10.11648/j.ijovs.20200501.11
}

Received: September 1, 2019; Accepted: October 16, 2019; Published: January 4, 2020

\begin{abstract}
The aim of the current research was to investigate the effect of visual fatigue induced with different clinical test stimuli on the sensory ocular dominance in dyslexic adults with Mearles-Irlen syndrome (MIS) and examine the role of colored filters. Nine female participants with dyslexia plus MIS aged between 18 and 36 years were recruited. Sensory ocular dominance and binocular vision functions were measured before and after induction of visual fatigue was induced. Measurements were conducted with participants wearing their habitual colored filters and without the filters and in two different sessions. Constant and continuous (static) test stimuli were used to create binocular rivalry. To induce visual fatigue, participants were asked to read, copy, and search for thirty minutes; the participants performed each tasks for ten minutes, while wearing their habitual near spectacle lens. No breaks were allowed between tasks. The calculated visual discomfort score (VDCS) for each test condition was analyzed. In sessions one and two respectively, VDCS was increased from $0.67 \pm 1.00$ to $1.44 \pm 0.53(p=0.07)$ and $0.22 \pm 0.44$ to $1.56 \pm 0.73(p=0.001)$, in adults with dyslexia and MIS after assessment of binocular vision function. Similarly, the VDCS was significantly increased to $2.56 \pm 0.73(p=0.002)$ in session one and $2.11 \pm 0.93(p=$ 0.001 ) in session two, after visual fatigue was induced in these participants. There were no differences between both eyes' sensory ocular dominance in dyslexic adults with MIS irrespective of the test stimuli used to induce the binocular rivalry. Induction of visual fatigue was followed by disruptions in the sensory ocular dominance that persisted with participants wearing their habitual colored overlays during the fatigue duration. This finding was also not influenced by the test slides used in these participants. Optometric investigations such as binocular vision testing, performed in dyslexic adults with MIS using certain stimuli could induce visual discomfort, but the discomfort induced by stressful visual testing was significantly greater. Moreover, the use of certain test stimuli was shown to disrupt the sensory ocular dominance of this group of participants suggesting that researchers should consider using the appropriate stimuli when examining patients with dyslexia and/or MIS and those experiencing reading difficulties.
\end{abstract}

Keywords: Dyslexia, Mearles-Irlen Syndrome, Colored Filters, Ocular Dominance, Visual Stress, Fatigue

\section{Introduction}

Dyslexia is defined as a specific learning disability that is characterized by difficulties with accurate and/or fluent word recognition and poor spelling and decoding abilities [1]. Mearles-Irlen syndrome (MIS) is a common neurological dysfunction that affects perceptual processing [2]. It can be an inherited genetic condition [2] or can occur as a result of a significant head trauma $[3,4]$. It is characterized by reports of visual perceptual distortions, typically when reading, that are alleviated by using colored filters [5]. In one study, MIS was reported to affect between 5 and $20 \%$ of the general population [6] and occurs in 30 to $45 \%$ of individuals with other conditions that are associated with learning difficulty, including dyslexia, photosensitive epilepsy, attention deficit hyperactivity disorder (ADHD), autism spectrum disorder (ASD), binocular instability and chronic fatigue syndrome $[7,8]$. 
Visual distortions such as blurriness, transposition, reversal, shimmer and glare, eyestrain and headaches have been shown to occur in people with MIS when reading texts or when observing striped patterns $[9,10]$. This is because MIS is thought to be driven by two independent dysfunctions within the brain. The dysfunction in the high-temporalresolution magnocellular pathways may destabilize the visual fixation (focus), resulting in the visual perceptual distortions $[4,11]$. Moreover, the attenuation of the inhibitory mechanisms in the hyper excitable visual cortex via visual stimulation could result in visual distortions of blurriness and glare $[12,13]$.

MIS is often misdiagnosed as dyslexia, however, there are distinct differences [14]. First, in dyslexia, the affected person experiences difficulties with comprehension when reading, which are associated with difficulties in sequencing letters and phonics. In contrast, a person with MIS experiences difficulty in word perception. Second, while a child with dyslexia can be trained in phonics and adjusting to their disability, a person with MIS will often require tinted glasses or colored overlays as a lifelong intervention [14] to alleviate the symptoms of visual distortions and discomfort, and improve reading accuracy $[3,6,10,15]$.

Binocular rivalry is used to provide a quantitative measure of sensory ocular dominance [16, 17]. It is obtained by calculating the difference in the exclusive visibility of rival stimuli between fellow eyes or between the dominant and the non-dominant eye $[18,19]$. When both eyes of a person are equally dominant, the exclusive visibility periods of rival stimuli are distributed equally $(50 \%)$ between fellow eyes. If the exclusive visibility of a given rival stimulus is longer ( $>$ $50 \%$ of rivalry time) than the perceived stimulus in the fellow eye, the eye that perceived the target for the longer duration becomes the dominant eye, while the fellow eye becomes the non-dominant eye $[18,19]$. Previous studies suggested that the visual confusion experienced by people with dyslexia may be caused by incomplete sensory dominance or confused cerebral dominance [20], which is further complicated by binocular retinal rivalry.

Although sensory visual input evidently plays a significant part in reading, there is no consensus in published literature on sensory ocular dominance and the effect of fatigue on its stability and duration in the dyslexic population with MIS. Previous studies that investigated the association between instability and motor ocular dominance, used the Dunlop test, and reading task $[11,21]$ and enrolled only children with dyslexia. While some authors suggested a causal relationship between dyslexia and instability of ocular dominance [11], others found that the test was less useful and the responses were variable suggesting [21] that the test is "unreliable.

The purpose of this study was to investigate the nature of binocular rivalry as a measure of sensory dominance in participants with dyslexia and MIS. The specific aims were as follows: (a) to investigate equal sensory dominance in dyslexic people with MIS and assess the stability of dominance duration; (b), to investigate the effects of induced visual fatigue on the quantity (duration) and quality (stability) of equi-dominance and cumulative exclusive visibility duration; and (c), to evaluate the effect of colored filters on visual discomfort and dominance duration and stability. Information obtained from this study, which is expected to provide data for the effect of visually induced fatigue on the quantity and quality of sensory ocular dominance and that for the effect of colored filters in the degree of visual comfort score (VCS) after visually induced fatigue, may be useful for the detection of MIS during optometric clinical investigation.

\section{Materials and Methods}

\subsection{Subjects}

Nine female students with dyslexia aged between 18 to 36 years were recruited over a 3-month period through fliers and posters distributed across Cardiff University. Participants who indicated interest were contacted via email. Only participants with an official diagnosis (statement) of dyslexia with MIS, which was defined by their use of color filters to help with reading, were included in this study. Participants who had a history of migraine, severe headache, photosensitive epilepsy, squint, and those who showed unequal visual acuity with a difference of more than two lines in logMAR chart between fellow eyes were excluded. All participants consented to participate in this study after the protocol was fully explained to them. Ethical approval was obtained from Cardiff University Ethical Committee, and all procedures followed the tenets of the Declaration of Helsinki (2008).

The participants were required to complete an extended set of survey questions that also contained the contact details of the investigators. Participants were required to attend the Optometry Clinic on two separate occasions that were at least one week apart. For each participant, approximately two hours of ocular examination was required for each visit and a questionnaire was administered on the first visit, to rule out any symptoms of asthenopia or a history of migraine. Preliminary examination included best corrected distance visual acuity (logMAR) measurements and stereo-acuity testing with the Titmus fly and cover test. For assessing ocular dominance, the hole in the card test was performed: an observer viewed a distant target through a small hole in the middle of a card. By participants closing either eye in turn or drawing hands towards their faces, the participants would be able to determine which eye the target was viewed through [22].

A VCS assessment was used before testing binocular rivalry and binocular vision functions. To examine the possibility that simply carrying out optometric investigations could induce visual discomfort in dyslexic persons, another VCS assessment was conducted. This was the pre-fatigue VCS assessment. Visual fatigue was then induced as described later and the following variables were assessed again: VCS, binocular rivalry, and binocular functions. This was the post-fatigue assessment. On the second visit, except for the use of participants' habitual filter (overlays or 
spectacles) during the fatigue session, testing followed a similar protocol as in the first visit.

\subsection{Sensory Ocular Dominance Testing}

The procedure for creating binocular rivalry using constant and continuous (static) test stimuli in this study was based on previous study that described and validated the technique [23]. The test stimuli were either a fixed high contrast (100\%) square wave grating that was presented in orthogonal directions and tilted $45^{\circ}$ to the right and $135^{\circ}$ to the left (as seen by the right and left eyes of the participants, respectively), or a fixed high contrast letters " $\mathrm{A}$ " and " $\mathrm{S}$ " that were presented to the right and left eyes, respectively. The gratings were presented as circular apertures with subtending fovea angles of $1^{\circ}$ and $2.5^{\circ}$ and diameters of $0.26 \mathrm{~cm}$ and $0.65 \mathrm{~cm}$, respectively. The circular aperture of field size $1^{\circ}$ gratings had spatial frequencies of either $2 \mathrm{c} / \mathrm{d}$ or $4 \mathrm{c} / \mathrm{d}$, and the letters were rival patterns. The $2.5^{\circ}$ field size grating had spatial frequencies of either $2 \mathrm{c} / \mathrm{d}$ or $4 \mathrm{c} / \mathrm{d}$ and the word stimulus "WAS" was presented to the right eye while the word stimulus "SAW" was presented to the left eye. A total of six sets of stimulus patterns were presented to each participant, and each stimulus had an identical $3.0 \times 3.0 \mathrm{~cm}$ (30\% grey) shaped fusion contour that was surrounded by a black distinct border and a transparent field.

Table 1 presents the stimuli field sizes and the rival patterns accordingly. Figure 1 shows the stimuli sizes, patterns and angles. The outlines of the squares/contours were used to maintain constant eye vergence and improve the stability and accuracy of the binocular alignment of the two rival targets [24].

Table 1. Target stimuli used in the study.

\begin{tabular}{|c|c|c|c|c|c|c|}
\hline Field size & $1^{\circ}$ & & & $2.5^{\circ}$ & & \\
\hline (slide) & (A) & (I) & (G) & (D) & (C) & $(\mathrm{H})$ \\
\hline Stimuli pattern & $2 \mathrm{c} / \mathrm{d}$ & $4 \mathrm{c} / \mathrm{d}$ & letters & $2 \mathrm{c} / \mathrm{d}$ & $4 \mathrm{c} / \mathrm{d}$ & words \\
\hline
\end{tabular}
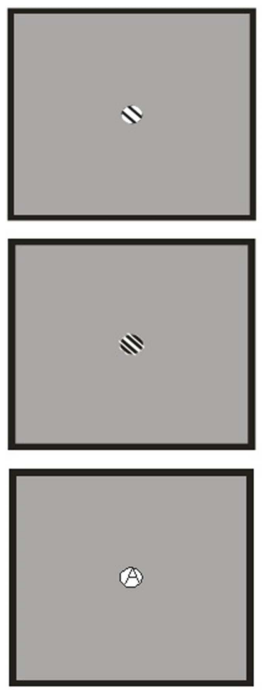
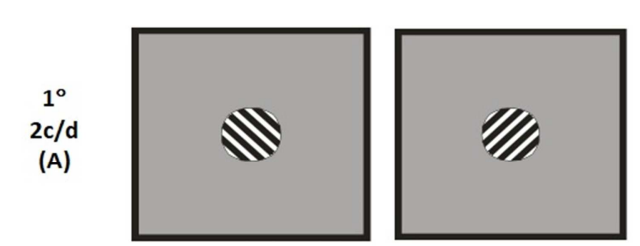

$2.5^{\circ}$

$2 \mathrm{c} / \mathrm{d}$

(D)

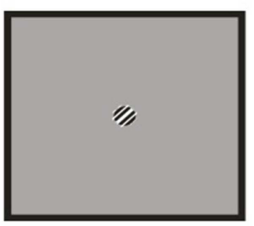

$1^{\circ}$

(I)
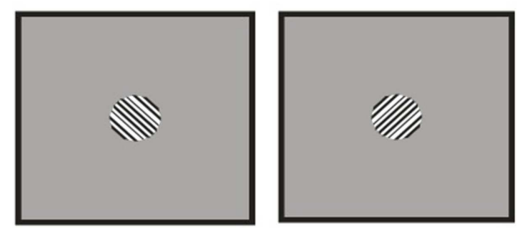

$2.5^{\circ}$

(C)
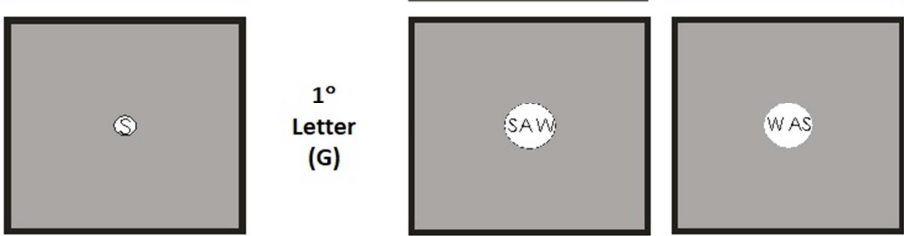

$2.5^{\circ}$

Word

Letter

Figure 1. Target stimuli sizes and angles.

In this study, rival stimuli targets that subtended a small retinal image size were used in order to minimize the piecemeal effect that has been reported previously $[25,26]$. The stimuli were drawn in black using a computer programming software (Corel DRAWx3 Version 13) and printed on a transparent sheet. Each pattern was adjusted so that it was located concentrically with a synoptophore slide of diameter $8.2 \mathrm{~cm} \times 8.2 \mathrm{~cm}$ and background luminance of 26 lux (measured at the level of eyepieces of the Synoptophore).

A synoptophore (Figure 2) was used to display the stimuli because it ensured binocular fixation and fusion while permitting independent control of test stimuli presentation for each eye. In any one trial, participants were presented with a pair of gratings with opposite orientations but with similar sizes and spatial frequencies. Background luminance of the two slides was equalized and the built-in rheostat adjusted the instrument to the highest intensity.

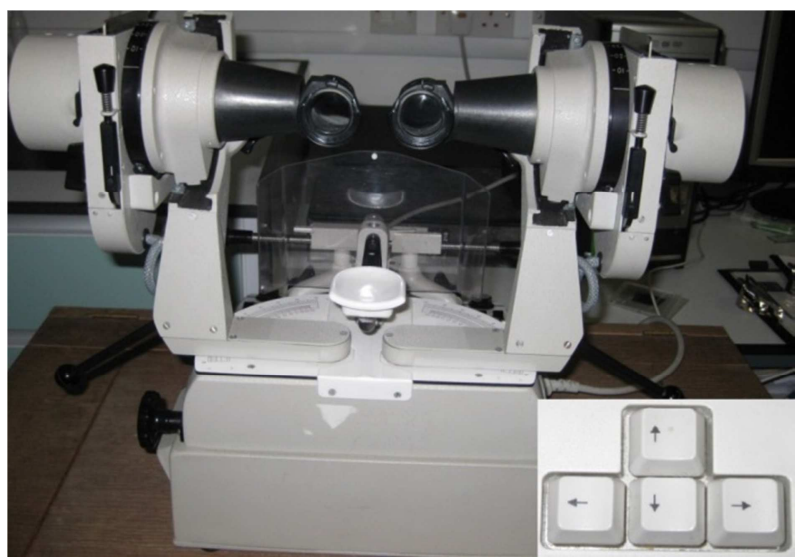

Figure 2. Synoptophore showing the keyboard arrows. 
Participants provided their responses for the right or the left image dominance (exclusive visibility) by pressing the rightward or leftward arrows of a computer keyboard placed on a table situated to the right side of the participants. Mixed responses (when the two images overlapped) were recorded when participants pressed the downward or upward arrows of the keyboard during testing.

\subsection{Procedure}

The experiment was conducted in a darkened room (illuminance approximately $5 \mathrm{lux}$ ), to reduce the incidence of the piecemeal effect $[25,27]$. For each participant, the synoptophore was aligned with their inter-pupillary distances and the chin rested on adjustable chin-rest. The test stimuli were viewed through the eyepieces of the synoptophore and the targets were aligned such that the participants were able to fuse the right and left stimuli. Participants rested the middle and index fingers of the right hand on the right and left arrow buttons on the keyboard to indicate the time of exclusive visibility of the rival target (for right eye or left eye responses). The fluctuations (changes) in the dominance of the two rival targets were manually recorded through the pair of buttons on the keyboard ("R" or "L"). Perceptual responses were obtained by pressing and releasing one of two keys consistently to indicate exclusive visibility of either eye's image (i.e. when the target was defined to be entirely dominant) and participants were asked to press the bottom (or the top) button when perceptual image was doubtful.

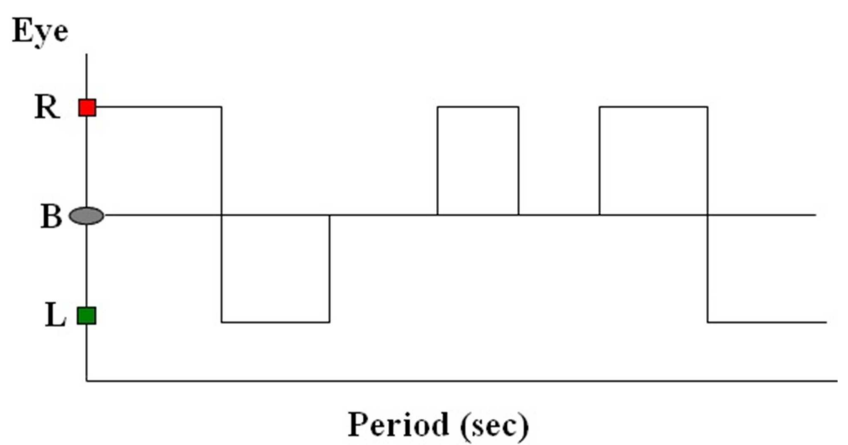

Figure 3. An example of participant responses during a trial of rivalry; $R$ (right eye), L (left eye), B (both eyes).

During rivalry conditions, participants' responses on perceptual grating dominancy were based on the grating that appeared to be covering the stimulus, i.e., exclusive visibility, rather than the relative clarity of the gratings. For example, participants pressed the right button when the rightward tilted (at $45^{\circ}$ ) grating, the letter "A" or the word "WAS", was exclusively visible over the field area. In contrast, they pressed the left button when the leftward tilted (at 135 ) gratings, the letter "S" or the word "SAW" was exclusively visible over the field area. The bottom key was pressed when both gratings, letters and words, were incompletely visible or overlapped (ambiguous perceptual response). The report of a dominance state was represented and shown in one of three responses: right eye dominance, left eye dominance or mixed dominance, i.e., when perceptual response represented pieces of either eye stimulus making the images of both eyes to appear overlapped. Figure 3 was an example of participant responses with both eyes during a trial.

Six presentations were shown to each participant on the same day, after a trial presentation was conducted with slide "A" to familiarize them with the test. The rivalry stimulus (slide A) was repeated during the experiment. Presentation of slides was carried out in random orders including the two sizes and stimuli patterns that were assigned to the two eyes. Each trial lasted for 70 seconds to ensure that rivalry would occur and that adequate time was given for measurement of ocular dominance. Each trial was followed by an inter-trial interval of about 45-60 seconds.

\subsection{Visual Fatigue Testing}

With their habitual corrections for near work, participants spent 10 minutes on each exercise including reading, copying, and searching, with no breaks between tasks. The reading text consisted of four simple, short and familiar French stories that were written in English language and selected from Google. The reading material was mounted on a standing clipboard that was tilted $35^{\circ}$ from the working table to ensure that the light was equally distributed. The material was placed $20 \mathrm{~cm}$ from the eyes and participants maintained the reading distance while pointing to the text lines with their fingers to avoid losing or jumping lines.

For the copying task, participants were asked to copy (with handwriting) a text passage (typed in French) onto a blank paper which was located $20 \mathrm{~cm}$ away from the eyes. The text passage was placed on a table or clipboard located $35 \mathrm{~cm}$ from the eyes. For the searching task, participants had to search through the text for four or five selected letters (e.g., s, v, x, z, and o) and recorded the number of times each letter was displayed and repeated within the text. For instance, letter "s" was repeated 123 times, "v" 13 times, "x" 4 times, "z" 3 times, and "o" was repeated 113 times, within a text of 25 lines with 340 closely spaced words written in Spanish. All tasks were performed under the same visual conditions: unusually close working distance $(20 \mathrm{~cm})$, low room illumination (40 lux), and small (10-point print size) bolded print superimposed on a visually stressful background stimulus. Figure 4 shows a sample of the stimulus reading material which consisted of an equally wide, black and white, horizontally striped alternating pattern. The print materials were closely spaced with bolded letters that were written in a 10-point Arial font. Visual discomfort was evaluated before and after performing the visual fatigue tasks using the visual comfort scale which ranked from the numbers " 0 " (comfortable) to "4" (uncomfortable tasks). 


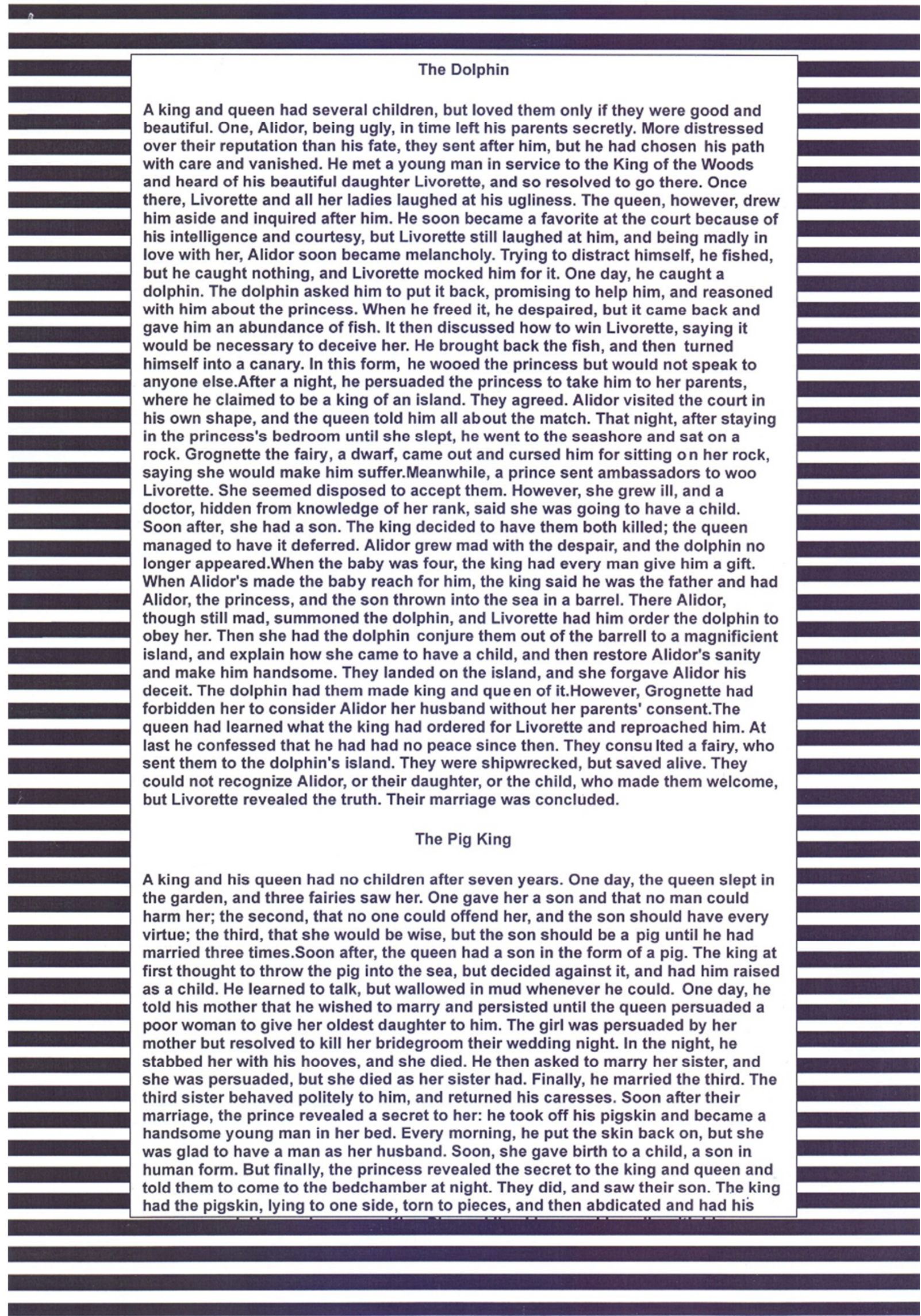

Figure 4. An example of stimulus reading material. 


\subsection{Statistical Analysis}

Quantitative assessment of time dominance was calculated as the total duration of exclusive target visibility viewed by a single eye and/or both eye images during a trial. Participant responses were stored in a software computer program. The strength of the ocular dominance and stability were indicated by the presence of a significant difference between duration, in terms of the number of seconds of exclusive visibility of a trial target for one eye compared with the other eye's target. The trial duration for all participants was revised to 60 seconds for each pair of slides used, i.e., 108 trials were revised (54 trials in each visit), and the numbers of switches for the right and left eye responses were computed in Microsoft Excel. The features that were calculated from the data included the cumulative exclusive rivalry duration (CERD; duration of exclusive visibility of the right eye image plus duration of exclusive visibility of the left eye image); mixed response duration (MRD; duration for which the participant sees pieces of either eye images - piecemeal); and cumulative exclusive rivalry rate (CERR; number of switch responses during cumulative exclusive rivalry duration). The binocular rivalry analysis targeted the CERD, the mixed response duration MRD, and the CERR. Paired t-test was used to compare between the CERD and MRD and the right and left eye exclusive visibility of rivalry duration. One way repeated measures analysis of variance was used to evaluate the effect of binocular vision $\mathrm{BV}$ tests and induced fatigue (with and without filters) on VCS in both visits; and to evaluate the effect of the rivalry stimulus pattern, field size, and special frequency on CERD and on the rivalry rate (RR) during cumulative exclusive rivalry duration [RR]. Analysis was conducted using SPSS (version 16, SPSS Inc., Chicago, Illinois, USA) statistical program. A $p$ value $<0.05$ was considered significant.

\section{Results}

\subsection{Mean Scores}

Of the nine dyslexic students with MIS, seven were right eye dominant $(77.8 \%)$ and two were left eye dominant $(22.2 \%)$, as tested with the hole in the card. The students used either colored overlays ( $n=6,66.7 \%)$ or tinted lenses $(n=3,33.3 \%)$ when reading. The mean refraction of the participants was $-0.08 \pm 0.18 \mathrm{D}$ and $-0.11 \pm 0.33 \mathrm{D}$, for the right and left eyes, respectively, and the frequency of the colors were in the order of blue $(5,55.6 \%)$, mint green, pink, violet, and yellow (one, $11.1 \%$ each). Table 2 shows the mean scores for sensory eye dominance in the fellow eyes. The mean sensory eye dominance scores which is shown in Table 2 were similar in the fellow eyes of dyslexic adults with MIS irrespective of the stimuli (i.e. slides) used ( $p \geq 0.2$, for all slides).

A comparison of the stability of sensory eye dominance with and without filters for the same visit (i.e. pre-fatigue and post-fatigue) showed significant differences for three of the six slides (i.e. slides $\mathrm{C}, \mathrm{D}, \& \mathrm{G}$ ), but the significant differences were consistent, i.e., they occurred in both visits, and only for slide "C" (

Switches refer to the number of participant responses per slide pair on an occasion and indicates the instability in participants' responses for either eye's image per slide pattern (response from exclusive right image to exclusive left image or to mixed response, i.e., merged image seen by both eyes simultaneously).

Table 2. Mean scores \pm standard deviation (SD) of sensory eye dominance $(n=9)$ for six test slides in visit one (pre-fatigue no filter visit).

\begin{tabular}{lll}
\hline Test slide & Eye & Mean \pm SD \\
\hline \multirow{2}{*}{ Pair 1 } & ARED1V1 & $20.2 \pm 10.3$ \\
& ALED1V1 & $22.5 \pm 10.8$ \\
Pair 2 & CRED1V1 & $19.1 \pm 8.8$ \\
& CLED1V1 & $20.4 \pm 6.8$ \\
Pair 3 & DRED1V1 & $17.8 \pm 8.5$ \\
& DLED1V1 & $19.7 \pm 8.3$ \\
Pair 4 & GRED1V1 & $15.8 \pm 8.3$ \\
& GLED1V1 & $15.7 \pm 7.3$ \\
Pair 5 & HRED1V1 & $19.0 \pm 14.9$ \\
& HLED1V1 & $10.6 \pm 11.1$ \\
Pair 6 & IRED1V1 & $19.8 \pm 8.5$ \\
& ILED1V1 & $22.3 \pm 8.4$ \\
\hline
\end{tabular}

ALED = left eye duration of exclusive visibility for slide $\mathrm{A} ; \mathrm{CRED}=$ right eye duration of exclusive visibility for slide $\mathrm{C}$; DRED $=$ right eye duration of exclusive visibility for slide $\mathrm{D}$; GRED = right eye duration of exclusive visibility for slide $\mathrm{G}$; HRED = right eye duration of exclusive visibility for slide $\mathrm{H}$; IRED = right eye duration of exclusive visibility for slide I. VI = visit 1 .

Table 3. Pre and post-fatigue comparison of mean scores for cumulative exclusive rivalry switches (CERS) in visits one and two (without V1 and with V2 filters, respectively) for all slides $(A, C, D, G, H$, and I).

\begin{tabular}{|c|c|c|c|c|c|}
\hline \multirow{2}{*}{ Slide } & \multirow{2}{*}{ Visit } & \multirow{2}{*}{ Mean difference \pm SD } & \multicolumn{2}{|c|}{$95 \%$ CI of the mean difference } & \multirow{2}{*}{$p$-Value } \\
\hline & & & Upper & Lower & \\
\hline Pair 1 & ACERS1V1 - ACERS2V1 & $-1.4 \pm 6.8$ & -6.6 & 3.8 & 0.54 \\
\hline Pair 2 & ACERS1V2 - ACERS2V2 & $3.0 \pm 4.3$ & -0.3 & 6.3 & $0.07 *$ \\
\hline Pair 3 & CCERS1V1 - CCERS2V1 & $7.2 \pm 6.4$ & 2.3 & 12.2 & $0.01 *$ \\
\hline Pair 4 & CCERS1V2 - CCERS2V2 & $6.1 \pm 4.7$ & 2.5 & 9.7 & $0.00 *$ \\
\hline Pair 5 & DCERS1V1 - DCERS2V1 & $5.2 \pm 4.4$ & 1.8 & 8.6 & $0.01 *$ \\
\hline Pair 6 & DCERS1V2 - DCERS2V2 & $1.2 \pm 6.1$ & -3.4 & 5.9 & 0.56 \\
\hline Pair 8 & GCERS1V2 - GCERS2V2 & $0.2 \pm 5.8$ & -4.2 & 4.7 & 0.91 \\
\hline Pair 9 & HCERS1V1 - HCERS2V1 & $1.7 \pm 5.1$ & -2.3 & 5.6 & 0.36 \\
\hline Pair 10 & HCERS1V2 - HCERS2V2 & $-0.1 \pm 1.8$ & -1.5 & 1.2 & 0.86 \\
\hline Pair 11 & ICERS1V1 - ICERS2V1 & $1.0 \pm 6.3$ & -3.9 & 5.9 & 0.65 \\
\hline Pair 12 & ICERS1V2 - ICERS2V2 & $-1.2 \pm 2.2$ & -2.9 & 0.4 & 0.13 \\
\hline
\end{tabular}

* Indicates significant difference. Slides are A, C, D, G, H \& I; CER = cumulative exclusive rivalry, S1 switches without fatigue, S2 switches with fatigue. 


\subsection{Effect of Fatigue (with and Without Colored Filters) on VCS}

Table 4 shows the results of one-way repeated-measures ANOVA assessing the effect of clinical binocular vision measurement and induced visual fatigue (with stressful reading material), without a color filter (visit one, V1) and with a color filter (visit two, V2) during the fatigue duration, on the VCS and visual analogue scale (VAS) scores. The post-hoc analysis comparing each of the two visits is presented in Table 5.

Table 4. Descriptive and comparative statistics of the effects of visual fatigue on visual comfortable score (VCS) and visual analogue scale (VAS) scores using repeated measures analysis of variance.

\begin{tabular}{lllll}
\hline Visit & Occasion & VCS (Mean \pm SD) & $\boldsymbol{p}$-Value & VAS (Mean \pm SD) \\
\hline \multirow{2}{*}{1} & Pre-fatigue & $0.67 \pm 1.00$ & & $1.59 \pm 2.04$ \\
& Post-BV tests & $1.44 \pm 0.53$ & $0.03^{*}$ & $3.18 \pm 0.92$ \\
& Post-fatigue & $2.56 \pm 0.73$ & & $5.96 \pm 2.02$ \\
\multirow{2}{*}{2} & Pre-fatigue & $0.22 \pm 0.44$ & & $0.02^{*}$ \\
& Post-BV tests & $1.56 \pm 0.73$ & $0.001^{*}$ & $3.83 \pm 1.58$ \\
& Post-fatigue & $2.11 \pm 0.93$ & & $5.30 \pm 1.97$ \\
\hline
\end{tabular}

* Indicates significant difference. $\mathrm{BV}=$ binocular vision.

Table 5. Post-hoc analysis comparing between visual comfort scores (VCS) throughout the fatigue process and the visual analogue scale (VAS) scores.

\begin{tabular}{llll}
\hline Visit & Occasion & P value VCS & P value VAS \\
\hline \multirow{4}{*}{1} & Pre-fatigue/ Post-BV tests & 0.07 & 0.19 \\
& Post-BV tests/ Post-fatigue & $0.02^{*}$ & $0.01^{*}$ \\
& Pre-fatigue /Post-fatigue & $0.02^{*}$ & $0.03^{*}$ \\
& Pre-fatigue/ Post-BV tests & $0.001^{*}$ & $0.001^{*}$ \\
2 & Post-BV tests/ Post-fatigue & 0.42 & 0.25 \\
& Pre-fatigue /Post-fatigue & $0.001^{*}$ & $0.001^{*}$ \\
\hline
\end{tabular}

* Indicates significant difference. $\mathrm{BV}=$ binocular vision.

There were significant differences between the fatigue procedures used in both visits for $\operatorname{VCS}(p=0.03$, fatigue without using colored filter for visit one; $p \quad 0.001$, fatigue with colored filters in place for visit 2$)$ and VAS ( $p=0.02$ and 0.001 , for visits one and two, respectively). Post-hoc analysis revealed that the post-reading fatigue process was the source of the significance differences in VCS and VAS scores found in visit 1 but not in visit 2, where the postbinocular vision test was the source of significant difference in VCS. No significant difference was found for the postreading process in visit 2 . For the VAS measure, similar results were found as for VCS, indicating that the use of colored filters significantly reduces the fatigue symptoms experienced by participants with MIS.

\subsection{Effect of Visual Fatigue (with and Without Color Filter) on Sensory Ocular Dominance and CERD in Dyslexic Adults with MIS}

The CERD and MRD were significantly correlated in both pre-fatigue ( $p$ 0.0001) visits, irrespective of the slide used. However, the mean pre-fatigue scores without filter with slide I were significantly higher for CERD than MRD (mean difference in scores, $95 \%$ confidence interval $=24.2,47.1$ to $1.3 ; \mathrm{t}=-2.4, \mathrm{df}=8, p=0.04)$ but approached significance for pair $1(25.4,51.1$ to $0.2 ; \mathrm{t}=-2.2, \mathrm{df}=8, p=0.05)$. For visit 2 , slide A also showed a significant difference $(p=0.03)$ while the other comparisons were not significantly different (Table $6)$.

Table 6. Comparison of the pre-fatigue results between CERD and mix responses (BED, both eyes response duration) in visits one and two.

\begin{tabular}{llll}
\hline Slide & Visit & Mean difference \pm SD & 95\% CI of the mean difference \\
\hline Pair 1 & ABED1V1 - ACERD1V1 & $-25.4 \pm 33.4$ & -51.6 to +0.2 \\
Pair 2 & ABED1V2 - ACERD1V2 & $-24.1 \pm 26.9$ & -44.7 to -3.4 \\
Pair 3 & CBED1V1 - CCERD1V1 & $-19.1 \pm 26.3$ & -39.3 to +1.1 \\
Pair 4 & CBED1V2 - CCERD1V2 & $-17.0 \pm 24.8$ & -36.0 to +2.1 \\
Pair 5 & DBED1V1 - DCERD1V1 & $-15.0 \pm 32.3$ & -39.8 to +9.9 \\
Pair 6 & DBED1V2 - DCERD1V2 & $-8.4 \pm 26.3$ & -28.5 to +11.8 \\
Pair 7 & GBED1V1 - GCERD1V1 & $-3.0 \pm 27.7$ & -24.3 to +18.3 \\
Pair 8 & GBED1V2 - GCERD1V2 & $-11.7 \pm 31.6$ & -34.0 to +12.6 \\
Pair 9 & HBED1V1 - HCERD1V1 & $+0.8 \pm 37.7$ & -28.2 to +29.7 \\
Pair 10 & HBED1V2 - HCERD1V2 & $-1.7 \pm 38.6$ & -31.4 to +28.0 \\
Pair 11 & IBED1V1 - ICERD1V1 & $-24.2 \pm 29.8$ & -0.07 \\
Pair 12 & IBED1V2 - ICERD1V2 & $-20.4 \pm 32.2$ & -47.1 to -1.3 \\
\hline
\end{tabular}

* Indicates significant difference. Slides are A, C, D, G, H, and I; CERD = cumulative exclusive rivalry duration, V1 without filter visit, V2 with filter visit.

Table 7. Mean scores \pm standard deviation (SD) of cumulative (right + left) exclusive rivalry duration (ERD) for all slides pre- and post-fatigue in visits 1 and 2.

\begin{tabular}{|c|c|c|c|c|}
\hline Slide & Visit & Mean difference \pm SD & $95 \%$ CI of the mean difference & $p$-Value \\
\hline Pair 1 & $\begin{array}{l}\text { ACERD1V1 } \\
\text { ACERD2V1 }\end{array}$ & $\begin{array}{l}42.7 \pm 16.7 \\
36.9 \pm 16.0\end{array}$ & -0.0 to +11.7 & 0.05 \\
\hline Pair 2 & ACERD1V2 & $42.0 \pm 13.4$ & -1.0 to +14.3 & 0.08 \\
\hline
\end{tabular}




\begin{tabular}{|c|c|c|c|c|}
\hline Slide & Visit & Mean difference \pm SD & $95 \%$ CI of the mean difference & $p$-Value \\
\hline \multirow{3}{*}{ Pair 3} & ACERD2V2 & $35.4 \pm 17.2$ & \multirow{3}{*}{+5.0 to +16.5} & \multirow{3}{*}{$0.00^{*}$} \\
\hline & CCERD1V1 & $39.5 \pm 13.1$ & & \\
\hline & CCERD2V1 & $28.8 \pm 12.7$ & & \\
\hline \multirow{2}{*}{ Pair 4} & CCERD1V2 & $38.5 \pm 12.4$ & \multirow{2}{*}{+2.4 to +12.5} & \multirow{2}{*}{$0.01 *$} \\
\hline & CCERD2V2 & $31.0 \pm 14.5$ & & \\
\hline \multirow{2}{*}{ Pair 5} & DCERD1V1 & $37.5 \pm 16.2$ & \multirow{2}{*}{-0.4 to +8.4} & \multirow{2}{*}{0.07} \\
\hline & DCERD2V1 & $33.5 \pm 14.0$ & & \\
\hline \multirow{2}{*}{ Pair 6} & DCERD1V2 & $35.1 \pm 12.4$ & \multirow{2}{*}{-3.4 to +7.1} & \multirow{2}{*}{0.45} \\
\hline & DCERD2V2 & $33.3 \pm 14.7$ & & \\
\hline \multirow{2}{*}{ Pair 7} & GCERD1V1 & $31.5 \pm 13.9$ & \multirow{2}{*}{-6.2 to +5.9} & \multirow{2}{*}{0.94} \\
\hline & GCERD2V1 & $31.7 \pm 15.2$ & & \\
\hline \multirow{2}{*}{ Pair 8} & GCERD1V2 & $35.8 \pm 15.8$ & \multirow{2}{*}{-1.4 to +8.8} & \multirow{2}{*}{0.13} \\
\hline & GCERD2V2 & $32.2 \pm 15.4$ & & \\
\hline \multirow{2}{*}{ Pair 9} & HCERD1V1 & $29.6 \pm 18.8$ & \multirow{2}{*}{-2.3 to +17.7} & \multirow{2}{*}{0.11} \\
\hline & HCERD2V1 & $21.9 \pm 14.4$ & & \\
\hline \multirow{2}{*}{ Pair 10} & HCERD1V2 & $30.9 \pm 19.3$ & \multirow{2}{*}{-2.7 to +22.2} & \multirow{2}{*}{0.11} \\
\hline & HCERD2V2 & $21.1 \pm 14.8$ & & \\
\hline \multirow{2}{*}{ Pair 11} & ICERD1V1 & $42.1 \pm 14.9$ & \multirow{2}{*}{+4.9 to +12.4} & \multirow{2}{*}{$0.00 *$} \\
\hline & ICERD2V1 & $33.5 \pm 14.0$ & & \\
\hline \multirow{2}{*}{ Pair 12} & ICERD1V2 & $40.2 \pm 16.1$ & \multirow{2}{*}{-5.2 to +10.9} & \multirow{2}{*}{0.44} \\
\hline & ICERD2V2 & $37.3 \pm 10.1$ & & \\
\hline
\end{tabular}

$\mathrm{A}=$ slide $\mathrm{A}, \mathrm{C}=$ cumulative, $\mathrm{E}=$ exclusive, $\mathrm{RD}=$ rivalry duration, $1=$ pre-fatigue, $\mathrm{V} 1=$ visit 1 (visit when fatigue was induced without using the filter).

The mean scores for CERD shown in Table 7 indicate significant differences for slides $\mathrm{C}$ and $\mathrm{I}$, which were consistent for slide $\mathrm{C}$ pre- and post-fatigue for slide $\mathrm{C}$ (without filters, $\mathrm{t}=4.3, \mathrm{df}=8, p=0.003$ and $\mathrm{t}=3.4$, $\mathrm{df}=8, p$ $=0.009$, for with filters), but significant differences were found for slide I, when participants did not use their filters $(\mathrm{t}$ $=5.4, \mathrm{df}=8, p=0.001$; Table 7).

\subsection{Effect of the Slides on the RR During Cumulative Exclusive Rivalry Duration (CERD)}

One way repeated measures ANOVA showed no significant difference in RR among rival stimuli used in the studied group $\left(\mathrm{F}_{5,4}=3.4 ; p=0.13\right)$ for the same visit. However, the least number of switches during the CERD occurred with slide $\mathrm{H}$. The number of switches made during CERD with slide $\mathrm{C}$ were significantly higher than with slide $\mathrm{H}(+11.3,+1.8$ to $+20.8 ; p=0.02)$. There was a borderline significance for the number of switches during CERD between slides $\mathrm{H}$ and I $(+10.0,-0.3$ to $+20.3 ; p=0.06)$ and between slides A and C $(5.4,+0.18$ to $+11.1 ; p=0.06)$. There was no other significant difference in the RR for the remainder of the slide comparisons.

Assessing the effect of different rivalry patterns on the MRD using one-way repeated measures ANOVA showed significant differences in MRD among the rivalry stimuli (slides) used $\left(\mathrm{F}_{5,4}=8.8, p=0.03\right)$. Slide $\mathrm{H}$ produced more mixed responses than the other slides, followed by slide $\mathrm{G}$. Post hoc analysis with Bonferroni correction showed differences only between slides $\mathrm{G}$ and $\mathrm{I}(+10.6,+1.4$ to +19.8 ; $p=0.02)$ and between slides $\mathrm{G}$ and $\mathrm{C}(+8.1,+0.7$ to $+15.5 ; p$ $=0.03$ ) for MRD. No significant difference was found between the other slides. The effect of fatigue (without, V1 and with, V2 filter) on the RR (number of switches) during both eyes rivalry duration is shown in Table 8 . Overall, fatigue caused an increase in the RR of participants who used colored filters but this was significant only when slide I was used (See pair 12, Table 8).

Table 8. The effect of fatigue (without, V1 and with, V2 filter) on the rivalry rate (number of switches) during both eyes rivalry duration.

\begin{tabular}{lllll}
\hline Slide & Visit & Mean difference \pm SD & $\mathbf{9 5 \%}$ CI of the mean difference & $\boldsymbol{p}$-Value \\
\hline Pair 1 & ABES1V1 - ABES2V1 & $-2.6 \pm 3.7$ & -5.4 to +0.3 & 0.07 \\
Pair 2 & ABES1V2 - ABES2V2 & $-1.6 \pm 4.5$ & -5.0 to +1.9 & 0.33 \\
Pair 3 & CBES1V1 - CBES2V1 & $-0.9 \pm 5.6$ & -5.2 to +3.4 & 0.65 \\
Pair 4 & CBES1V2 - CBES2V2 & $-0.1 \pm 4.9$ & -3.9 to +3.6 & 0.95 \\
Pair 5 & DBES1V1 - DBES2V1 & $0.2 \pm 3.3$ & -2.3 to +2.8 & 0.85 \\
Pair 6 & DBES1V2 - DBES2V2 & $1.2 \pm 3.6$ & -1.6 to +4.0 & 0.34 \\
Pair 7 & GBES1V1 - GBES2V1 & $0.7 \pm 4.3$ & -2.6 to +4.0 & 0.65 \\
Pair 8 & GBES1V2 - GBES2V2 & $-1.3 \pm 4.1$ & -4.5 to +1.8 & 0.36 \\
Pair 9 & HBES1V1 - HBES2V1 & $-1.8 \pm 4.1$ & -4.9 to +1.3 & 0.23 \\
Pair 10 & HBES1V2 - HBES2V2 & $-1.1 \pm 1.5$ & -2.2 to +0.0 & 0.05 \\
Pair 11 & IBES1V1 - IBES2V1 & $-1.6 \pm 4.9$ & -5.3 to +2.2 & 0.36 \\
Pair 12 & IBES1V2 - IBES2V2 & $-5.1 \pm 3.5$ & -7.8 to -2.4 & $0.00^{*}$ \\
\hline
\end{tabular}

\section{Discussion}

This study investigated the effect of induced visual fatigue on sensory ocular dominance and the influence of colored filters on the levels of visual fatigue in dyslexic adults with MIS. The findings showed that sensory ocular dominance 
was similar between the fellow eyes of dyslexic adults with MIS and this was independent of the test stimuli used to induce rivalry. When participants were subjected to stressful reading conditions using three test stimuli (slides $\mathrm{C}, \mathrm{D}$ and $\mathrm{G})$, the sensory ocular dominance became unstable and remained so even when the participants wore their colored overlays. For one test stimuli (slide C), there was instability in the sensory dominance of participants with and without their colored filters. These findings indicate that visual fatigue disrupted the sensory ocular dominance of adults with dyslexia and MIS, regardless of whether they used colored overlays. In addition, some test stimuli disrupted the sensory ocular dominance of participants with dyslexia and MIS while others maintained the sensory ocular dominance, suggesting the need for practitioners to select the proper test stimuli for clinical testing of adults with dyslexia and MIS.

The current study showed that the use of colored overlays was associated with a significant reduction in the symptoms of visual fatigue reported by adults with dyslexia and MIS, thus confirming their beneficial effects in relieving visual discomfort in participants [15]. A double-masked controlled study demonstrated that by using colored overlays to aid with reading, participants experienced a reduction in the frequency of eyestrain and headache in comparison with the findings obtained when not using colored overlays [15]. This beneficial effect was shown to persist and was not attributable to placebo effects [28]. Colored overlays have been largely employed to remedy for some aspects of the reading difficulties experienced by dyslexic individuals $[21,29]$. When applied above written texts, colored overlays positively influence both reading fluency and reading speed [3, 15, 30]. These effects are particularly evident for individuals with MIS, who experience eyestrain and/or visual distortions while reading $[31,32]$.

With the slide $\mathrm{H}$, which was the $2.5^{\circ}$ (WAS/SAW) rivalry stimulus, participants showed longer MRDs than CERDs, indicating that the presence of the central " $A$ " acted as a "fusion lock". This finding is in line with the suggestion that inter-ocular differences in visual attributes that are predominantly processed in the parvocellular pathway leads to rivalry, and that differences in visual attributes that are predominantly processed in the magnocellular pathway tend to integrate [33]. The results showed a trend of longer MRDs in dyslexia with MIS, showing the reduced automatic focusing of visual attention in dyslexic adults which was related to deficit of the magnocellular system [34-36]. Another explanation is that it may be due to the equidominance interpreting the sensory visual inputs with visual confusion in the dyslexic subjects.

Another significant finding of this study was the quality of equi-dominance in terms of the stability of dominance (amount of switching) in dyslexic adults. Adults with dyslexia and MIS exhibited substantial instability in their responses. When the participants were tested with slide $\mathrm{H}$ (WAS/SAW stimuli), they showed fewer switches, suggesting that they had a more stable response. One explanation for this finding could be the delay in selection due to the visual confusion that occurs between either the presented stimuli image in one (dominant) hemisphere and the memory image in the other (non-dominant) hemisphere. This then leads to confusion in the word sequence orientation or sequence [20]. Excessive sensory input switching in MIS dyslexia adults may be attributable to cortical hyper excitability of their sensory visual system [37], which probably led to the unstable image they reported.

Although, this study may be limited by the lack of a control group and the small sample size, two other studies on MIS had only a study group [14, 31]. The small sample size was because the participants were only volunteers who responded to the invitation that they were willing to undertake the long clinical tests of visual fatigue induction over the two visits.

\section{Conclusion}

The findings showed that slides A and I had longer cumulative exclusive rivalry durations and fewer switches (more stability) during post-fatigue testing of ocular dominance stability in dyslexic participants with MIS, suggesting that these slides are suitable for measurement in these participants. Slides $\mathrm{G}$ and $\mathrm{H}$ provoked a greater proportion of mixed rivalry and are important slides for use in dyslexic adults. While slide $\mathrm{G}$, which contains letters, tests the parvocellular system, slide $\mathrm{H}$ reflects the magnocellular function. Simply carrying out optometric investigations with certain stimuli could induce visual discomfort in dyslexic persons and researchers should consider this when examining similar populations or those with reading difficulties. The findings further highlight the need for professional eye care for people with suspected dyslexia plus Meares-Irlen syndrome.

\section{Acknowledgements}

The author thanks King Saud University for the financial support.

\section{Conflict of Interest}

The authors do not have any possible conflicts of interest.

\section{References}

[1] Lyon GR, Shaywitz SE, Shaywitz BAJAod. A definition of dyslexia. JSTOR 2003; 53: 1-14.

[2] Taub MB, Shallo-Hoffmann J, Steinman S, Steinman B. The effect of colored overlays on reading eye movements in adults. J. Behav. Optom. 2009; 20: 143-149.

[3] Wilkins AJ, Evans BJ. Visual stress, its treatment with spectral filters, and its relationship to visually induced motion sickness. Appl. Ergon. 2010; 41: 509-515.

[4] Evans BJW, Wilkins AJ, Brown J, Busby A, Wingfield A, Jeanes $\mathrm{R}$ et al. A preliminary investigation into the aetiology of Meares-Irlen syndrome. Ophthalmic Physiol. Opt. 1996; 16: 286-296. 
[5] Evans BJW. The need for optometric investigation in suspected Meares-Irlen syndrome or visual stress. Ophthalmic Physiol. Opt. 2005; 25: 363-370.

[6] Kriss I, Evans BJW. The relationship between dyslexia and Meares-Irlen syndrome. J. Res. Read. 2005; 28: 350-364.

[7] Wilkins A, Allen PM, Monger LJ, Gilchrist JM. Visual stress and dyslexia for the practicing optometrist. Optom. Practice 2016; 17: 103-112.

[8] Chouinard BD, Zhou CI, Hrybouski S, Kim ES, Cummine J. A functional neuroimaging case study of Meares-Irlen syndrome/visual stress (MISViS). Brain Topogr. 2012; 25: 293-307.

[9] Evans BJW. Dyslexia and Vision: Wiley; Whurr Publishers Ltd, Londion, UK, 2001.

[10] Irlen H. Scotopic sensitivity/Irlen syndrome: hypothesis and explanation of the syndrome. J. Behav. Optom. 1994; 5: 65-66.

[11] Stein JF, Fowler S. Diagnosis of dyslexia by means of a new indicator of eye dominance. British J. Ophthalmol. 1982; 66: 332-336.

[12] Wilkins A, Huang J, Cao Y. Visual stress theory and its application to reading and reading tests. J. Res. Read. 2004; 27: $152-162$.

[13] Huang J, Cooper TG, Satana B, Kaufman DI, Cao Y. Visual distortion provoked by a stimulus in migraine associated with hyperneuronal activity. Headache 2003; 43: 664-671.

[14] Chang M, Kim S-H, Kim J-Y, Cho YA. Specific visual symptoms and signs of Meares-Irlen syndrome in Korean. Korean J. Ophthalmol. 2014; 28: 159-163.

[15] Wilkins AJ, Evans BJ, Brown JA, Busby AE, Wingfield AE, Jeanes RJ et al. Double-masked placebo-controlled trial of precision spectral filters in children who use coloured overlays. Ophthalmic Physiol. Opt. 1994; 14: 365-370.

[16] Walls GL. A theory of ocular dominance. AMA Arch. Ophthalmol. 1951; 45: 387-412.

[17] Porac C, Coren S. Lateral Preferences and Human Behavior: Springer-Verlag: New York, USA, 1981.

[18] Handa T, Mukuno K, Uozato H, Niida T, Shoji N, Shimizu K. Effects of dominant and nondominant eyes in binocular rivalry. Optom. Vis. Sci. 2004; 81: 377-383.

[19] Ooi TL, He ZJ. Sensory eye dominance. Optometry 2001; 72: $168-178$.

[20] Orton ST. Word-blindness in school children. Archiv. Neurol. Psychiatry 1925; 14: 581-615.

[21] Evans BJ, Drasdo N, Richards IL. Investigation of accommodative and binocular function in dyslexia. Ophthalmic Physiol. Opt. 1994; 14: 5-19.
[22] Mendola JD, Conner IP. Eye dominance predicts fMRI signals in human retinotopic cortex. Neurosci. Lett. 2007; 414: 30-34.

[23] Alanazi MA. Studies of Visual Functions and the Effect of Visual Fatigue in Adults with Dyslexia: PhD Thesis, Cardiff University: UK, 2010.

[24] Wiesenfelder H, Blake R. The neural site of binocular rivalry relative to the analysis of motion in the human visual system. J. Neurosci. 1990; 10: 3880-3888.

[25] O'Shea RP, Blake R, Wolfe JM. Binocular rivalry and fusion under scotopic luminances. Perception 1994; 23: 771-784.

[26] Freeman AW, Nguyen VA. Controlling binocular rivalry. Vis. Res. 2001; 41: 2943-2950.

[27] Bonneh Y, Sagi D, Karni A. A transition between eye and object rivalry determined by stimulus coherence. Vis. Res. 2001; 41: 981-989.

[28] Bouldoukian J, Wilkins AJ, Evans BJ. Randomised controlled trial of the effect of coloured overlays on the rate of reading of people with specific learning difficulties. Ophthalmic \& Physiol. Opt. 2002; 22: 55-60.

[29] Aasved H. Ophthalmological status of school children with dyslexia. Eye 1987; 1: 61-68.

[30] Uccula A, Enna M, Mulatti C. Colors, colored overlays, and reading skills. Front. Psychol. 2014; 5: 833.

[31] Imaizumi S, Hibino H, Koyama S. Effect of colored overlays on reading comfort in people with and without Meares-Irlen syndrome. Int. J. Affect. Eng. 2016; 15: 21-28.

[32] Alanazi MA, Alanazi SA, Osuagwu UL. Evaluation of visual stress symptoms in age-matched dyslexic, Meares-Irlen syndrome and normal adults. Int. J. Ophthalmol. 2016; 9: 617-624.

[33] Carlson TA, He S. Visible binocular beats from invisible monocular stimuli during bivocular rivalry. Curr. Biol. 2000; 10: $1055-1058$.

[34] Facoetti A, Lorusso ML, Paganoni P, Cattaneo C, Galli R, Mascetti GG. The time course of attentional focusing in dyslexia and normally reading children. Brain and Cogn. 2003; 53: $181-184$

[35] Skottun BC, Skoyles JR. Attention, reading and dyslexia. Clin. Exp. Optom. 2006; 89: 241-245.

[36] Boden C, Giaschi D. M-Stream deficits and reading related visual processes in developmental dyslexia. Psychol. Bull. 2007; 133: 346-366.

[37] Wilkins AJ. Visual Stress. Oxford Psychology Series, Oxford University Press: New York, NY, US, 1995. 